\title{
The Suslinian number and other cardinal invariants of continua
}

by

\section{T. Banakh (Kielce and Lviv), V. V. Fedorchuk (Moscow), J. Nikiel (Opole) and M. Tuncali (North Bay)}

\begin{abstract}
By the Suslinian number $\operatorname{Sln}(X)$ of a continuum $X$ we understand the smallest cardinal number $\kappa$ such that $X$ contains no disjoint family $\mathcal{C}$ of non-degenerate subcontinua of size $|\mathcal{C}|>\kappa$. For a compact space $X, \operatorname{Sln}(X)$ is the smallest Suslinian number of a continuum which contains a homeomorphic copy of $X$. Our principal result asserts that each compact space $X$ has weight $\leq \operatorname{Sn}(X)^{+}$and is the limit of an inverse well-ordered spectrum of length $\leq \operatorname{Sln}(X)^{+}$, consisting of compacta with weight $\leq \operatorname{Sln}(X)$ and monotone bonding maps. Moreover, $w(X) \leq \operatorname{Sln}(X)$ if no $\operatorname{Sln}(X)^{+}$-Suslin tree exists. This implies that under the Suslin Hypothesis all Suslinian continua are metrizable, which answers a question of Daniel et al. [Canad. Math. Bull. 48 (2005)]. On the other hand, the negation of the Suslin Hypothesis is equivalent to the existence of a hereditarily separable non-metrizable Suslinian continuum. If $X$ is a continuum with $\operatorname{Sln}(X)<2^{\aleph_{0}}$, then $X$ is 1-dimensional, has rim-weight $\leq \operatorname{Sln}(X)$ and weight $w(X) \geq \operatorname{Sln}(X)$. Our main tool is the inequality $w(X) \leq \operatorname{Sln}(X) \cdot w(f(X))$ holding for any light map $f: X \rightarrow Y$.
\end{abstract}

In this paper we introduce a new cardinal invariant related to the Suslinian property of continua. By a continuum we understand any compact connected Hausdorff space. Following Lelek [7], we define a continuum $X$ to be Suslinian if it contains no uncountable family of pairwise disjoint nondegenerate subcontinua. The simplest example of a Suslinian continuum is the usual interval $\mathbb{I}=[0,1]$. On the other hand, the existence of nonmetrizable Suslinian continua is a subtle problem. The properties of such continua were considered in [1]. It was shown in [1] that each Suslinian continuum $X$ is perfectly normal, rim-metrizable, and 1-dimensional. Moreover, a locally connected Suslinian continuum has weight $\leq \omega_{1}$.

The simplest examples of non-metrizable Suslinian continua are Suslin lines. However this class of examples has a consistency flavor since no Suslin

2010 Mathematics Subject Classification: Primary 54F15; Secondary 54C05, 54F05, 54F50. Key words and phrases: Suslinian continua, Suslinian number, inverse limits, locally connected continuum, light mappings. 
line exists in some models of ZFC (for example, in models satisfying (MA+ $\neg \mathrm{CH})$ ). It turns out that any example of a non-metrizable locally connected Suslinian continuum necessarily has consistency nature: the existence of such a continuum is equivalent to the existence of a Suslin line (see [1]). This implies that under the Suslin Hypothesis (asserting that no Suslin line exists) each locally connected Suslinian continuum is metrizable.

It is clear that each Suslinian continuum $X$ has countable Suslin number $c(X)$. At this point we recall the definition of some known topological cardinal invariants. Given a topological space $X$ let

- $c(X)=\sup \{|\mathcal{U}|: \mathcal{U}$ is a disjoint family of non-empty open subsets of $X\}$ be the Suslin number of $X$;

- $l(X)=\min \{\kappa$ : each open cover of $X$ contains a subcover of size $\leq \kappa\}$ be the Lindelöf number of $X$;

- $d(X)=\min \{|D|: D$ is a dense set in $X\}$ be the density of $X$;

- $h l(X)=\sup \{l(Y): Y \subset X\}$ be the hereditary Lindelöf number of $X$;

- $h d(X)=\sup \{d(Y): Y \subset X\}$ be the hereditary density of $X$;

- $w(X)=\min \{|\mathcal{B}|: \mathcal{B}$ is a base of the topology of $X\}$ be the weight of $X$;

- $\operatorname{rim}-w(X)=\min \left\{\sup _{U \in \mathcal{B}} w(\partial U): \mathcal{B}\right.$ is a base of the topology of $\left.X\right\}$ be the rim-weight of $X$.

In the context of Suslinian continua, by analogy with the Suslin number $c(X)$ it is natural to introduce a new cardinal invariant

$\operatorname{Sln}(X)=\sup \{|\mathcal{C}|: \mathcal{C}$ is a disjoint family of non-degenerate subcontinua of $X\}$

defined for any continuum $X$ and called the Suslinian number of $X$. Thus a continuum $X$ is Suslinian if and only if $\operatorname{Sln}(X) \leq \aleph_{0}$.

It is clear that $\operatorname{Sln}(X) \leq \operatorname{Sln}(Y)$ for any pair $X \subset Y$ of continua. It will be convenient to extend the definition of $\operatorname{Sn}(X)$ to all Tikhonov spaces letting

$$
\operatorname{Sln}(X)=\min \{\operatorname{Sln}(Y): Y \text { is a continuum containing } X\}
$$

for a Tikhonov space $X$.

Like many other cardinal invariants the Suslinian number is monotone.

Proposition 1. If $X$ is a Tikhonov space and $Y$ is a subspace of $X$, then $\operatorname{Sln}(Y) \leq \operatorname{Sln}(X)$.

The cardinal invariant $\operatorname{Sn}(X)$ is not trivial since it can attain any infinite value.

Proposition 2. $\operatorname{Sln}(X)=c(X)=w(X)=\kappa$ for the hedgehog $X=$ $\left\{\left(x_{\alpha}\right)_{\alpha<\kappa}:\left|\left\{\alpha<\kappa: x_{\alpha} \neq 0\right\}\right| \leq 1\right\} \subset[0,1]^{\kappa}$ with $\kappa$ needles. 
Note that each hedgehog is rim-finite in the sense that it has a base of the topology consisting of sets with finite boundaries. Let us remark that a rim-finite continuum $X$ with uncountable Suslinian number must be non-metrizable (because rim-countable metrizable continua are Suslinian, see [7]).

The Suslinian number cannot increase under monotone maps. We recall that a map $f: X \rightarrow Y$ is monotone if $f^{-1}(y)$ is connected for any $y \in Y$.

Proposition 3. If $X$ and $Y$ are compact spaces and $f: X \rightarrow Y$ is a surjective monotone map, then $\operatorname{Sln}(Y) \leq \operatorname{Sln}(X)$.

Proof. Embed $X$ in a continuum $Z$ with $\operatorname{Sln}(Z)=\operatorname{Sln}(X)$. Consider the following equivalence relation on $Z: x \sim y$ if either $x=y$ or $x, y \in X$ and $f(x)=f(y)$. Let $T=Z / \sim$ be the quotient space and $q: Z \rightarrow T$ be the quotient map. Since all the equivalence classes are connected, the quotient map $q$ is monotone. Since the preimage of a connected set under a monotone map is connected, $\operatorname{Sn}(T) \leq \operatorname{Snn}(Z)$. It remains to observe that $Y$ can be identified with a subspace of $T$, which yields $\operatorname{Snn}(Y) \leq \operatorname{Snn}(T) \leq \operatorname{Sn}(Z)=$ $\operatorname{Sin}(X)$. For a similar argument, we also refer the readers to Theorem 2.4.13 in 3 .

Proposition 4. If $X$ is a Tikhonov space and $K$ is a compact subset of $X$, then $\operatorname{Sln}(X / K) \leq \operatorname{Sln}(X)$.

Proof. Let $Z$ be a continuum that contains $X$ and has $\operatorname{Sln}(Z)=\operatorname{Sln}(X)$. Since $K$ is a compact subspace of $X$, the quotient space $X / K$ naturally embeds into the quotient space $Z / K$. We claim that $\operatorname{Sln}(Z / K) \leq \operatorname{Sln}(Z)$. In the opposite case we would find a disjoint family $\mathcal{C}$ of subcontinua in $Z / K$ having the cardinality $|\mathcal{C}|>\operatorname{Sln}(Z)$. At most one subcontinuum $C \in \mathcal{C}$ can contain the point $K \in Z / K=\{K\} \cup(Z \backslash K)$. Deleting this subcontinuum from the family $\mathcal{C}$, if necessary, we can assume that $\bigcup \mathcal{C} \subset Z \backslash K$. Then $\mathcal{C}$ can be thought of as a disjoint family of subcontinua of $Z$ having size $|\mathcal{C}|>\operatorname{Sln}(Z)$, which contradicts the definition of $\operatorname{Sln}(Z)$. This contradiction witnesses that $\operatorname{Sln}(Z / K) \leq \operatorname{Sln}(Z)$ and then

$$
\operatorname{Sln}(X / K) \leq \operatorname{Sln}(Z / K) \leq \operatorname{Sln}(Z)=\operatorname{Sln}(X)
$$

Recall that a map $f: X \rightarrow Y$ between compact Hausdorff spaces is called light if $f^{-1}(y)$ is zero-dimensional for each $y \in Y$.

Theorem 1. If $X$ and $Y$ are compact spaces and $f: X \rightarrow Y$ is a light map, then $w(X) \leq w(Y) \cdot \operatorname{Sln}(X)$.

For the proof of this theorem we shall need two lemmas.

LEMma 1. For any point $z$ of a continuum $Z$ there is a family $\mathcal{U}$ of closed neighborhoods of $z$ in $Z$ such that $|\mathcal{U}| \leq \operatorname{Sln}(Z)$ and $\bigcap \mathcal{U}$ is zero-dimensional. 
Proof. We shall construct a transfinite sequence $\left(U_{\alpha}\right)_{\alpha<\alpha_{0}}$ of closed neighborhoods of $z$ and a transfinite sequence $\left(K_{\alpha}\right)_{\alpha<\alpha_{0}}$ of pairwise disjoint, non-degenerate subcontinua of $Z$ such that $K_{\alpha} \subset \bigcap_{\beta<\alpha} U_{\beta}$ and $U_{\alpha} \cap K_{\alpha}=\emptyset$ for each $\alpha<\alpha_{0}$.

To start the construction we choose any subcontinuum $K_{0} \subset Z \backslash\{z\}$ and take any closed neighborhood $U_{0} \subset Z$ of $z$ missing the set $K_{0}$. Then $U_{0}$ is not zero-dimensional, and since $Z$ is a continuum, we can find a subcontinuum $K_{1} \subset U_{0}$ not containing the point $z$.

Suppose that for some ordinal $\alpha$ the closed neighborhoods $U_{\beta}, \beta<\alpha$, of $z$ are already selected so that $\bigcap_{\beta<\alpha} U_{\beta}$ is not zero-dimensional. Choose any non-degenerate continuum $K_{\alpha} \subset \bigcap_{\beta<\alpha} U_{\beta} \backslash\{z\}$. Then choose a closed neighborhood $U_{\alpha}$ of $z$ which is disjoint from $K_{\alpha}$. Observe that when $\beta<\alpha$, then $K_{\beta} \cap U_{\beta}=\emptyset$ and $K_{\alpha} \subset U_{\beta}$, whence $K_{\beta} \cap K_{\alpha}=\emptyset$.

The construction should stop at some ordinal $\alpha_{0}$ of size $\left|\alpha_{0}\right| \leq \operatorname{Sln}(Z)$. For this ordinal the intersection $\bigcap_{\alpha<\alpha_{0}} U_{\alpha}$ is zero-dimensional. Then $\mathcal{U}=$ $\left\{U_{\alpha}: \alpha<\alpha_{0}\right\}$ is the required family of closed neighborhoods of the point $z$ in $Z$.

Lemma 2. For any closed subset $K$ of a continuum $Z$ there is a family $\mathcal{U}$ of closed neighborhoods of $K$ such that $|\mathcal{U}| \leq \operatorname{Sln}(Z)$ and $\cap \mathcal{U} \backslash K$ is zerodimensional.

Proof. Consider the quotient space $Z / K=\{K\} \cup(Z \backslash K)$ of $Z$ by $K$ and let $q: Z \rightarrow Z / K$ be the quotient map. By Lemma 1, the continuum $Z / K$ contains a family $\mathcal{V}$ of closed neighborhoods of the point $K \in Z / K$ such that $|\mathcal{V}| \leq \operatorname{Sln}(Z / K)$ and the intersection $\bigcap \mathcal{U}$ is zero-dimensional. It is easy to see that the family $\mathcal{U}=\left\{q^{-1}(V): V \in \mathcal{V}\right\}$ of closed neighborhoods of $K$ has the desired property: it has cardinality $|\mathcal{U}| \leq|\mathcal{V}| \leq \operatorname{Sln}(Z / K) \leq \operatorname{Sln}(Z)$ and $\bigcap \mathcal{U} \backslash K$ is zero-dimensional (being homeomorphic to $\bigcap \mathcal{V} \backslash\{K\}$ ).

Proof of Theorem 1. Let $f: X \rightarrow Y$ be a light map between compact Hausdorff spaces. We need to prove that the weight of $X$ satisfies $w(X) \leq \kappa$ where $\kappa=w(Y) \cdot \operatorname{Sn}(X)$. Let $Z$ be a continuum such that $Z \supset X$ and $\operatorname{Sln}(X)=\operatorname{Sln}(Z)$. Of course, $\operatorname{Sln}(Z) \leq \kappa$.

By Lemma 2 , the continuum $Z$ contains a family $\mathcal{U}$ of closed neighborhoods of the subset $X \subset Z$ such that $|\mathcal{U}| \leq \operatorname{Sln}(Z) \leq \kappa$ and $\bigcap \mathcal{U} \backslash X$ is zero-dimensional. The family $\mathcal{U}$ can be used to construct a map $g: Z \rightarrow \mathbb{I}^{\kappa}$ such that $X \subset g^{-1}(\mathbf{0}) \subset \bigcap \mathcal{U}$, where $\mathbf{0}=\{0\}^{\kappa} \in[0,1]^{\kappa}=\mathbb{I}^{\kappa}$. It follows that $g^{-1}(\mathbf{0}) \backslash X \subset \bigcap \mathcal{U} \backslash X$ is zero-dimensional.

Since $w(Y) \leq \kappa$, the space $Y$ can be identified with a subset of the Tikhonov cube $\mathbb{I}^{\kappa}$. It follows from the Tietze-Urysohn Theorem that the map $f$ can be extended to a map $\bar{f}: Z \rightarrow \mathbb{I}^{\kappa}$. Now consider the map

$$
h=(\bar{f}, g): Z \rightarrow \mathbb{I}^{\kappa} \times \mathbb{I}^{\kappa}, \quad z \mapsto(\bar{f}(z), g(z)),
$$


and observe that

$$
X \subset h^{-1}\left(\mathbb{I}^{\kappa} \times \mathbf{0}\right)=g^{-1}(\mathbf{0}) \subset \bigcap \mathcal{U}
$$

It follows that for every $y \in \mathbb{I}^{\kappa} \times \mathbf{0}$ the preimage $h^{-1}(y)$ lies in the union $f^{-1}(y) \cup(\bigcap \mathcal{U} \backslash X)$ of two zero-dimensional spaces and hence is zero-dimensional.

Since $Z$ is a continuum, each component of a non-empty open set $U$ contains a non-trivial subcontinuum. Consequently, $U$ has at most $\operatorname{Sln}(X)$ components. Denote by $\mathcal{C}_{U}$ the family of closures of components of $U$.

Let $\mathcal{B}$ be a base for the topology of $h(Z)$ with $|\mathcal{B}| \leq \kappa$. Finally consider the family $\mathcal{C}=\bigcup_{B \in \mathcal{B}} \mathcal{C}_{h^{-1}(B)}$ of closed subsets of $Z$, which has size at most $\kappa$. Because of the compactness of $X$, the inequality $w(X) \leq \kappa$ will follow as soon as we prove that the family $\mathcal{C}$ separates the points of $X$ in the sense that any two distinct points $x, y \in X$ lie in disjoint elements $C_{x}, C_{y}$ of the family $\mathcal{C}$.

If $h(x) \neq h(y)$, then we can find two basic subsets $B_{x}, B_{y} \in \mathcal{B}$ with disjoint closures such that $h(x) \in B_{x}$ and $h(y) \in B_{y}$. Let $D_{x}$ be the component of $h^{-1}\left(B_{x}\right)$, containing the point $x$ and $D_{y}$ be the component of $h^{-1}\left(B_{y}\right)$, containing the point $y$. Then $\bar{D}_{x}, \bar{D}_{y}$ are disjoint elements of $\mathcal{C}$ separating the points $x, y$.

Next, suppose that $h(x)=h(y)=z$ and observe that $z \in h(X) \subset \mathbb{I}^{\kappa} \times \mathbf{0}$. It follows from the zero-dimensionality of $\bigcap \mathcal{U} \backslash X$ and the inclusion $h^{-1}(z) \subset$ $f^{-1}(z) \cup(\bigcap \mathcal{U} \backslash X)$ that the set $h^{-1}(z)$ is zero-dimensional. Consequently, we can find two open subsets $O_{x}, O_{y} \subset Z$ with disjoint closures such that $x \in O_{x}, y \in O_{y}$ and $h^{-1}(z) \subset O_{x} \cup O_{y}$. Since the map $h$ is closed, there is a basic neighborhood $B_{z} \in \mathcal{B}$ of $z$ such that $h^{-1}\left(B_{z}\right) \subset O_{x} \cup O_{y}$. Then $x$ and $y$ lie in the closures of distinct components of $h^{-1}\left(B_{z}\right)$. This completes the proof that the collection $\mathcal{C}$ separates the points of $X$.

The previous theorem allows us to generalize the classical monotone-light Factorization Theorem [12, 13.3] asserting that any map $f: X \rightarrow Y$ between compact Hausdorff spaces can be represented as the (unique) composition $\lambda \circ \mu$ of a monotone map $\mu: X \rightarrow Z$ and a light map $\lambda: Z \rightarrow Y$. Applying the preceding theorem and two propositions to the calculation of the weight of the space $Z$, we conclude that $w(Z) \leq w(Y) \cdot \operatorname{Sln}(Z) \leq w(Y) \cdot \operatorname{Sln}(X)$. In this way we obtain the following corollary.

COROLlary 1. Let $f: X \rightarrow Y$ be a map between compact spaces and $f=\lambda \circ \mu$ be the monotone-light decomposition of $f$ into a monotone surjective map $\mu: X \rightarrow Z$ and a light map $\lambda: Z \rightarrow Y$. Then $w(Z) \leq w(Y) \cdot \operatorname{Sln}(X)$ and the non-degeneracy set $N_{\mu}=\left\{z \in Z:\left|\mu^{-1}(z)\right|>1\right\}$ of $\mu$ has size $\left|N_{\mu}\right| \leq \operatorname{Sln}(X)$. 
In this corollary, if we assume that $f$ is the constant map, then the space $Z$ is the decomposition of $X$ into its components and it is zero-dimensional. Thus we obtain the following corollary.

Corollary 2. Each compact Hausdorff space $X$ admits a monotone map $f: X \rightarrow Z$ onto a zero-dimensional space $Z$ of weight $w(Z) \leq \operatorname{Sln}(X)$. In particular, each zero-dimensional compact space $Z$ has weight $w(Z) \leq$ $\operatorname{Sln}(Z)$.

As another application of Theorem 1 we prove that each Suslinian continuum $X$ is hereditarily decomposable, that is, $X$ contains no indecomposable subcontinuum (a continuum $X$ is indecomposable if $X$ cannot be written as the union of two proper non-degenerate subcontinua of $X$ ).

Proposition 5. If $X$ is a Tikhonov space with $\operatorname{Sln}(X) \leq \aleph_{0}$, then all compact zero-dimensional subspaces of $X$ are metrizable and all subcontinua of $X$ are decomposable.

Proof. If $Z$ is a zero-dimensional compact subset of $X$, then $w(Z) \leq$ $\operatorname{Sln}(Z) \leq \operatorname{Sln}(X) \leq \aleph_{0}$ by the preceding corollary.

Now take any subcontinuum $C$ of $X$. Then $\operatorname{Sln}(C) \leq \operatorname{Sln}(X) \leq \aleph_{0}$, which means that the continuum $C$ is Suslinian. Let $f: C \rightarrow[0,1]$ be any nonconstant map. By Theorem 1, the map $f$ can be written as the composition $f=\lambda \circ \mu$ of a monotone map $\mu: C \rightarrow Z$ and a light map $\lambda: Z \rightarrow[0,1]$ of some continuum $Z$ with $w(Z) \leq \operatorname{Sln}(C) \leq \aleph_{0}$. Thus, $Z$ is a metrizable Suslinian continuum. Such a continuum is decomposable. Otherwise, since each indecomposable continuum has uncountably many composants (see [6, Theorem $7^{\prime}$, p. 213]), we would have $\operatorname{Sln}(Z)>\aleph_{0}$. Consequently, we can write $Z=A \cup B$ as the sum of two properly smaller subcontinua $A, B \subset Z$. Their preimages $\mu^{-1}(A)$ and $\mu^{-1}(B)$ under the monotone map $\mu$ are proper subcontinua of $C$ whose union equals $C$. This means that the continuum $C$ is decomposable.

Next we prove that the hereditary Lindelöf number of any space $X$ is bounded from above by the Suslinian number of $X$. For Suslinian continua this result was proved in Theorem 1 of $[1]$.

Theorem 2. $h l(X) \leq \operatorname{Sln}(X)$ for any Tikhonov space $X$.

Proof. Let $\kappa=\operatorname{Sln}(X)$ and $Z \supset X$ be a continuum with $\operatorname{Sln}(Z)=\kappa$.

First, we prove that each singleton $\left\{x_{0}\right\}, x_{0} \in X$, is the intersection of $\kappa$ many neighborhoods in $Z$. By Lemma 1, there is a family $\mathcal{N}$ of closed neighborhoods of $x_{0}$ in $Z$ such that $|\mathcal{N}| \leq \operatorname{Sln}(Z)=\kappa$ and the intersection $\bigcap \mathcal{N}$ is zero-dimensional. The compactum $Y=\bigcap \mathcal{N}$, being zerodimensional, admits a light map onto the singleton. Applying Theorem 1 , we get $w(Y) \leq \operatorname{Sln}(Y) \leq \operatorname{Sln}(Z)=\kappa$. Consequently, we can find a fam- 
ily $\mathcal{N}^{\prime}$ of neighborhoods of the point $x_{0}$ in $Z$ such that $Y \cap \bigcap \mathcal{N}^{\prime}=\left\{x_{0}\right\}$ and $\left|\mathcal{N}^{\prime}\right| \leq \kappa$. Then the family $\mathcal{N} \cup \mathcal{N}^{\prime}$ has size $\leq \kappa$ and its intersection is $\left\{x_{0}\right\}$.

Now, take any subspace $A \subset X$ and let $\mathcal{U}$ be a cover of $A$ by open subsets of $Z$. Then $\bigcup \mathcal{U}$ is an open subset of $Z$ and $B=Z \backslash \cup \mathcal{U}$ is a closed set in $Z$. Consider the quotient space $Z / B=(Z \backslash B) \cup\{B\}$ and let $q: Z \rightarrow Z / B$ be the quotient map. Since $\operatorname{Sn}(Z / B) \leq \operatorname{Sln}(Z)=\kappa$, we may apply the previous reasoning to find a family $\mathcal{V}$ of open neighborhoods of the singleton $\{B\} \in Z / B$ with $\{B\}=\bigcap \mathcal{V}$ and $|\mathcal{V}| \leq \kappa$. Then $\mathcal{W}=\left\{q^{-1}(V): V \in \mathcal{V}\right\}$ is a family of size $\leq \kappa$ with $\bigcap \mathcal{W}=B$. The complement $Z \backslash W$ of each $W \in \mathcal{W}$ is a compact subset of $Z$ which can be covered by a finite subcollection of $\mathcal{U}$. Therefore, the union $\bigcup_{W \in \mathcal{W}}(Z \backslash W)=\bigcup \mathcal{U}$ can be covered by $\leq \kappa$ elements of the cover $\mathcal{U}$.

According to [3, 3.12.10(1)], $w(X) \leq 2^{h l(X)}$ for any compact Hausdorff space. Hence, $w(X) \leq 2^{\operatorname{Sin}(X)}$ for any Tikhonov space. In fact, we shall prove a stronger upper bound $w(X) \leq \operatorname{Snn}(X)^{+}$.

The Generalized Suslin Hypothesis asserts that for any regular cardinal $\kappa$ there is no $\kappa$-Suslin tree, where a tree is called $\kappa$-Suslin if it has height $\kappa$ but contains no chain or antichain of length $\kappa$. We recall that the classical Suslin Hypothesis asserts that there is no $\aleph_{1}$-Suslin tree.

Below, for a cardinal $\kappa$, we denote by $\operatorname{cf}(\kappa)$ the cofinality of $\kappa$ and by $\kappa^{+}$ the successor cardinal of $\kappa$. We identify cardinals with initial ordinals.

Theorem 3. Let $X$ be a Tikhonov space. Then $w(X) \leq \operatorname{Sln}(X)^{+}$. Moreover, if no $\kappa^{+}$-Suslin tree exists for $\kappa=\operatorname{Sn}(X)$, then $w(X) \leq \operatorname{Sln}(X)$.

Proof. Let $\kappa=\operatorname{Sln}(X)$ and embed $X$ into a continuum $K$ with $\operatorname{Sln}(K)=$ $\operatorname{Sln}(X)$. Assuming that $\kappa^{+}<w(X) \leq w(K)$, we can find a continuous map $f: K \rightarrow Z$ of $K$ onto a continuum $Z$ of weight $w(Z)=\kappa^{++}$. Moreover, we may assume that the map $f$ is monotone. Indeed, if $f$ were not monotone, then it would factorize as $f=\lambda \circ \mu$ with $\mu: K \rightarrow Z_{1}$ monotone and $\lambda: Z_{1} \rightarrow Z$ light. Then $w\left(Z_{1}\right) \leq w(Z) \cdot \operatorname{S} \ln (K)=\kappa^{++} \cdot \kappa=\kappa^{++}$. Now, let us see that the conditions $\operatorname{Sln}(Z) \leq \kappa$ and $w(Z)=\kappa^{++}$lead to a contradiction.

Express $Z$ as the inverse limit of a well-ordered transfinite spectrum $\left\{Z_{\alpha}\right.$ : $\left.\alpha<\kappa^{++}\right\}$consisting of continua $Z_{\alpha}$ with $w\left(Z_{\alpha}\right) \leq \kappa^{+}$. Let $p_{\alpha}: Z \rightarrow Z_{\alpha}$, $\alpha<\kappa^{++}$, denote the (surjective) limit projections of the spectrum.

Consider the family $\mathcal{T}=\left\{p_{\alpha}^{-1}(z): z \in Z_{\alpha}, \alpha<\kappa^{++}, \operatorname{dim} p_{\alpha}^{-1}(z)>0\right\}$ of point-preimages which are not zero-dimensional. Endowed with the inverse inclusion order, this family forms a tree. This tree has no chains of length more than $\kappa$. Otherwise we would obtain a strictly decreasing sequence of length $>\kappa$ consisting of closed subsets of $Z$, which is impossible as $h l(Z) \leq$ $\operatorname{Sln}(Z)=\kappa$. 
The tree also contains no antichain of length $>\kappa$ since otherwise we would construct a disjoint family of size $>\kappa$ consisting of components of some elements of $\mathcal{T}$. Consequently, the tree $\mathcal{T}$ has height $\leq \kappa^{+}$and all levels of the tree have size $\leq \kappa$. This implies that the tree $\mathcal{T}$ contains at most $\kappa^{+}$ elements. Since $\kappa^{+}<\kappa^{++}=\operatorname{cf}\left(\kappa^{++}\right)$, we can find an ordinal $\alpha<\kappa^{++}$such that for any point $z \in Z_{\alpha}$ the preimage $p_{\alpha}^{-1}(z)$ is zero-dimensional. This means that the limit projection $p_{\alpha}: Z \rightarrow Z_{\alpha}$ is light. Applying Theorem 1. we get a contradiction: $w(Z) \leq w\left(Z_{\alpha}\right) \cdot \operatorname{Sln}(Z) \leq \kappa^{+}$.

If no $\kappa^{+}$-Suslin tree exists, then the tree $\mathcal{T}$ constructed above is not $\kappa^{+}$-Suslin and thus has height $\leq \kappa$. In this case we replace the condition $w(Z)=\kappa^{++}$by $w(Z)=\kappa^{+}$and see that the proof above gives that $w(Z) \leq$ $\operatorname{Sln}(Z)$.

Corollary 3. If the Generalized Suslin Hypothesis holds, then $w(X) \leq$ $\operatorname{Sln}(X)$ for any Tikhonov space $X$.

Applying Theorem 3 to Suslinian continua, we obtain the answer to the second part of Problem 1 of [1].

COROLlary 4. Under the Suslin Hypothesis all Suslinian continua are metrizable.

Theorem 3 allows us to describe the structure of compacta $X$ with $w(X)>\operatorname{Sln}(X)$.

TheOREM 4. Each compact space $X$ with $w(X)>\operatorname{Sln}(X)$ is the inverse limit of a well-ordered spectrum $\left\{Z_{\alpha}, \pi_{\alpha}^{\beta}, \alpha \leq \beta<\operatorname{Sn}(X)^{+}\right\}$consisting of compacta of weight $w\left(Z_{\alpha}\right) \leq \operatorname{Sn}(X)$ and monotone bonding maps $\pi_{\alpha}^{\beta}: Z_{\beta} \rightarrow Z_{\alpha}$.

Proof. Let $\kappa=\operatorname{Sln}(X)$. It follows from Theorem 3 that $w(X)=\kappa^{+}$. Therefore, we can write $X$ as the inverse limit of a well-ordered spectrum $\mathcal{S}=\left\{X_{\alpha}, p_{\alpha}^{\beta}, \alpha \leq \beta<\kappa^{+}\right\}$consisting of compacta of weight $\leq \kappa$ and surjective bonding maps. Since $h l(X) \leq \operatorname{Sn}(X) \leq \kappa$, this spectrum is factorizable in the sense that any continuous map $f: X \rightarrow Z$ into a compact space $Z$ of weight $w(Z) \leq \kappa$ can be written as a composition $f=f_{\alpha} \circ p_{\alpha}$ of the limit projection $p_{\alpha}: X \rightarrow X_{\alpha}$ and a continuous map $f_{\alpha}: X_{\alpha} \rightarrow Z$ for some ordinal $\alpha<\kappa^{+}$(see [5, 3.1.6]).

For each ordinal $\alpha<\kappa^{+}$let $p_{\alpha}=\lambda_{\alpha} \circ \mu_{\alpha}$ be the (unique) monotonelight decomposition of the limit projection $p_{\alpha}: X \rightarrow X_{\alpha}$ into a monotone map $\mu_{\alpha}: X \rightarrow Z_{\alpha}$ and a light map $\lambda_{\alpha}: Z_{\alpha} \rightarrow X_{\alpha}$. By Proposition 3 , $\operatorname{Sln}\left(Z_{\alpha}\right) \leq \operatorname{Sln}(X) \leq \kappa$ and by Theorem 1. $w\left(Z_{\alpha}\right) \leq w\left(X_{\alpha}\right) \cdot \operatorname{Sln}\left(Z_{\alpha}\right) \leq \kappa$. Then there is an ordinal $\xi(\alpha)>\alpha$ such that the monotone map $\mu_{\alpha}: X \rightarrow Z_{\alpha}$ factorizes through $X_{\xi(\alpha)}$ in the sense that $\mu_{\alpha}=\mu_{\alpha}^{\xi(\alpha)} \circ p_{\xi(\alpha)}$ for some map $\mu_{\alpha}^{\xi(\alpha)}: X_{\xi(\alpha)} \rightarrow Z_{\alpha}$ 
Thus we obtain the following commutative diagram:

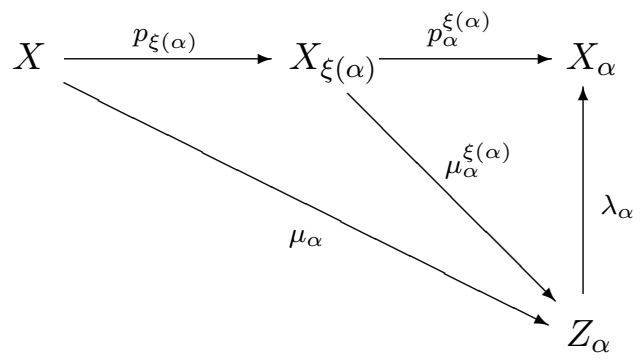

Let $A$ be a cofinal subset of ordinals $<\kappa^{+}$such that $\xi(\alpha)<\beta$ for any $\alpha<\beta$ in $A$. For any $\alpha<\beta$ in $A$ define a bonding map $\pi_{\alpha}^{\beta}: Z_{\beta} \rightarrow Z_{\alpha}$ letting $\pi_{\alpha}^{\beta}=\mu_{\alpha}^{\xi(\alpha)} \circ p_{\xi(\alpha)}^{\beta} \circ \lambda_{\beta}$. We claim that the map $\pi_{\alpha}^{\beta}$ is monotone. This follows from the monotonicity of the map $\mu_{\alpha}=\mu_{\alpha}^{\xi(\alpha)} \circ p_{\xi(\alpha)}^{\beta} \circ p_{\beta}=$ $\mu_{\alpha}^{\xi(\alpha)} \circ p_{\xi(\alpha)}^{\beta} \circ \lambda_{\beta} \circ \mu_{\beta}=\pi_{\alpha}^{\beta} \circ \mu_{\beta}$. Indeed, for any point $y \in Z_{\alpha}$, the preimage $\left(\pi_{\alpha}^{\beta}\right)^{-1}(y)=\mu_{\beta}\left(\mu_{\alpha}^{-1}(y)\right)$ is connected, being the image of the connected set $\mu_{\alpha}^{-1}(y)$.

It is easy to see that $\pi_{\alpha}^{\gamma}=\pi_{\alpha}^{\beta} \circ \pi_{\beta}^{\gamma}$ for any ordinals $\alpha<\beta<\gamma$ in $A$, which means that $\mathcal{S}^{\prime}=\left\{Z_{\alpha}, \pi_{\alpha}^{\beta}: \alpha, \beta \in A\right\}$ is an inverse spectrum. Let $Z=\lim \mathcal{S}^{\prime}$ be the limit of this spectrum. Observe that the monotone maps $\mu_{\alpha}: X \rightarrow Z_{\alpha}, \alpha \in A$, induce a surjective map $\mu: X \rightarrow Z$ while the light maps $\lambda_{\alpha}: Z_{\alpha} \rightarrow X_{\alpha}, \alpha \in A$, induce a surjective map $\lambda: Z \rightarrow X$. Since $\lambda_{\alpha} \circ \mu_{\alpha}=p_{\alpha}$ for all $\alpha \in A$, the composition $\lambda \circ \mu: X \rightarrow X$ is the identity map of $X$. Consequently, both $\lambda$ and $\mu$ are homeomorphisms and thus $X$ can be identified with the limit $Z$ of the spectrum $\mathcal{S}^{\prime}$ of length $\kappa^{+}$consisting of compacta of weight $\leq \kappa$ and monotone bonding maps.

The following particular case of Theorems 3 and 4 answers the remaining part of Problem 1 from [1].

COROLlary 5. Each non-metrizable Suslinian continuum $X$ has weight $\aleph_{1}$ and is the limit of an inverse spectrum of length $\aleph_{1}$ consisting of metrizable Suslinian continua and monotone bonding maps.

In the subsequent proof we shall refer to properties of the hyperspace $\exp (X)$ of a given compact Hausdorff space $X$. The hyperspace $\exp (X)$ of $X$ is the space of all non-empty closed subsets of $X$, endowed with the Vietoris topology. It is well known that $\exp (X)$ is a compact Hausdorff space with $w(\exp (X))=w(X)$. We denote by $\exp _{c}(X)$ the subspace of $\exp (X)$ consisting of subcontinua of $X$. It is easy to see that $\exp _{c}(X)$ is a closed subspace in $\exp (X)$. 
Compacta $X$ with small Suslinian number $\operatorname{Sln}(X)<\mathfrak{c}$ share many properties of Suslinian continua.

Theorem 5. If $X$ is a continuum with $\operatorname{Sln}(X)<\mathfrak{c}$, then $\operatorname{dim} X \leq 1$ and $\operatorname{rim}-w(X) \leq \operatorname{Sln}(X) \leq h l\left(\exp _{c}(X)\right) \leq w(X) \leq \operatorname{Sln}(X)^{+}$.

Proof. Let $\kappa=\operatorname{Sln}(X)$. To show that $\operatorname{rim}-w(X) \leq \operatorname{Sln}(X)$, take any point $x \in X$ and a neighborhood $U \subset X$ of $x_{0}$. Let $f: X \rightarrow[0,1]$ be any function with $f\left(x_{0}\right)=\{0\}$ and $f^{-1}([0,1)) \subset U$. Since $\operatorname{Sln}(X)<\mathfrak{c}$, the set $\left\{y \in(0,1): \operatorname{dim} f^{-1}(y)>0\right\}$ has size $\leq \operatorname{Sln}(X)<\mathfrak{c}$. Consequently, we can find a point $y \in(0,1)$ whose preimage $f^{-1}(y) \subset Z$ is zero-dimensional. By Corollary 2, $w\left(f^{-1}(y)\right) \leq \operatorname{Sln}\left(f^{-1}(y)\right) \leq \operatorname{Sln}(X)=\kappa$.

Now consider the neighborhood $V=f^{-1}([0, y))$ whose boundary $\partial V$ lies in $f^{-1}(y)$ and thus has weight $w(\partial V) \leq \kappa$ and is zero-dimensional. This proves the inequality $\operatorname{rim}-w(X) \leq \kappa$, and shows that the small inductive dimension of $X$ satisfies $\operatorname{ind}(X) \leq 1$. By [3, 7.2.7], $\operatorname{dim} X \leq 1$.

It remains to prove that $\kappa \leq h l\left(\exp _{c}(X)\right) \leq w(X) \leq \operatorname{Sln}(X)^{+}$. The third inequality was proved in Theorem 3 while the second inequality follows from $h l\left(\exp _{c}(X)\right) \leq w\left(\exp _{c}(X)\right) \leq w(\exp (X))=w(X)$. Assuming $h l\left(\exp _{c}(X)\right)$ $<\kappa=\operatorname{Sln}(X)$, let $\lambda=h l\left(\exp _{c}(X)\right)$ and find a disjoint family $\mathcal{C}$ of size $|\mathcal{C}|=\lambda^{+}$consisting of non-degenerate subcontinua of $X$. This family $\mathcal{C}$ can be considered as a subset of the hyperspace $\exp _{c}(X)$ of subcontinua of $X$. Identify $X$ with the set of all degenerate subcontinua in $\exp _{c}(X)$. Since $h l\left(\exp _{c}(X)\right)=\lambda$, the set $\mathcal{C}$ contains a subset $\mathcal{C}^{\prime}$ of size $\left|\mathcal{C}^{\prime}\right|=|\mathcal{C}|=\lambda^{+}$whose closure in $\exp _{c}(X)$ misses $X$.

We claim that $\mathcal{C}^{\prime}$ is not a scattered subspace of $\exp _{c}(X)$. Let us recall that a topological space is scattered if each of its subspaces has an isolated point. It is known (and can be easily shown) that the size of a scattered space is equal to its hereditary Lindelöf number. Since $\left|\mathcal{C}^{\prime}\right|=\lambda^{+}>\lambda=$ $h l\left(\exp _{c}(X)\right) \geq h l\left(\mathcal{C}^{\prime}\right)$, the space $\mathcal{C}^{\prime}$ is not scattered and thus contains a subspace $\mathcal{C}^{\prime \prime}$ having no isolated point.

Now we shall construct a subset $\left\{C_{t}\right\}_{t \in T} \subset \mathcal{C}^{\prime \prime}$ indexed by elements of the binary tree $T=\bigcup_{n \in \mathbb{N}}\{0,1\}^{n}$ as follows. The binary tree $T$ consists of finite binary sequences. Given two binary sequences $t=\left(t_{0}, \ldots, t_{n}\right), s=$ $\left(s_{0}, \ldots, s_{m}\right)$ in $T$ we write $t \leq s$ if $n \leq m$ and $t_{i}=s_{i}$ for all $i \leq n$.

Take any distinct elements $C_{0}, C_{1} \in \mathcal{C}^{\prime \prime}$ and observe that the subcontinua $C_{0}, C_{1}$ are disjoint (because the family $\mathcal{C}$ is disjoint). Hence, they have open neighborhoods $U_{0}, U_{1} \subset X$ with disjoint closures.

Assuming that for some binary sequence $s=\left(s_{0}, \ldots, s_{n}\right)$ the subcontinuum $C_{s} \in \mathcal{C}^{\prime \prime}$ and its neighborhood $U_{s} \subset X$ are constructed, consider the open subset $\mathcal{U}_{s}=\left\{C \in \mathcal{C}^{\prime \prime}: C \subset U_{s}\right\}$ of the space $\mathcal{C}^{\prime \prime}$ and take any two distinct (and hence disjoint) subcontinua $C_{s^{\wedge} 0}, C_{s^{\wedge} 1} \in \mathcal{U}_{s}$. Next, choose two 
open neighborhoods $U_{s^{\wedge} 0}, U_{s^{\wedge} 1} \subset U_{s}$ of $C_{s^{\wedge} 0}, C_{s^{\wedge} 1}$ with disjoint closures. This finishes the inductive step.

Now, for any infinite binary sequence $s=\left(s_{i}\right)$ let $C_{s}$ be a cluster point of the set $\left\{C_{(s \mid n)}: n \in \mathbb{N}\right\}$ in $\exp (X)$, where $s \mid n=\left(s_{0}, \ldots, s_{n-1}\right)$. It is easy to see that $\left\{C_{s}: s \in\{0,1\}^{\omega}\right\}$ is a disjoint family of subcontinua of $X$, lying in the closure of the set $\mathcal{C}^{\prime \prime}$. Since this closure misses the set $X$, each continuum $C_{s}, s \in\{0,1\}^{\omega}$, is non-degenerate. Thus, $\kappa=\operatorname{Sln}(X) \geq \mid\left\{C_{s}\right.$ : $\left.s \in\{0,1\}^{\omega}\right\} \mid=\mathfrak{c}$, which is a contradiction.

Problem 1. Is $\operatorname{rim}-w(X) \leq \operatorname{Sln}(X)$ for any compact Hausdorff space $X$ ?

Let us remark that all examples of non-metrizable Suslinian continua considered in the introduction or in [1] contain a copy of a Suslin line and hence fail to be hereditarily separable. However (consistent) examples of non-metrizable, hereditarily separable Suslinian continua can be constructed as well. For such a construction we need the following definitions and the lemma.

We recall that a surjective map $f: X \rightarrow Y$ is irreducible if $f(Z) \neq Y$ for any proper closed subset $Z$ of $X$. This is equivalent to saying that a set $D \subset X$ is dense in $X$ provided $f(D)$ is dense in $Y$.

Following [4, III.1.15] we call a monotone map $f: X \rightarrow Y$ between two continua atomic if for every non-degenerate subcontinuum $Z \subset Y$ the map $f \mid f^{-1}(Z): f^{-1}(Z) \rightarrow Z$ is irreducible. This is equivalent to saying that $\bar{D}=f^{-1}(\overline{f(D)})$ for every subset $D \subset X$ whose image $f(D)$ is dense in some non-degenerate subcontinuum of $Y$. An atomic map $f: X \rightarrow Y$ will be called $I$-atomic if for every $y \in Y$ the preimage is a singleton or an arc in $X$.

The following lemma will be our basic tool in the subsequent inductive construction.

Lemma 3. For any non-degenerate metrizable Suslinian continuum $Y$ and any countable set $Z \subset Y$ there are a metrizable Suslinian continuum $X$ and an I-atomic map $f: X \rightarrow Y$ whose non-degeneracy set $N(f)=\{y \in Y$ : $\left.\left|f^{-1}(y)\right|>1\right\}$ equals $Z$.

Proof. For every $z \in Z$ fix a decreasing neighborhood base $\left(O_{n}(z)\right)_{n \in \omega}$ at $z$ such that $\overline{O_{n+1}(z)} \subset O_{n}(z)$ for all $n \in \omega$. Let $\left\{q_{n}: n \in \omega\right\}$ be a countable dense set in $\mathbb{I}=[0,1]$. Fix a map $h_{z}: Y \backslash\{z\} \rightarrow \mathbb{I}$ such that $h_{z}\left(\partial O_{n}(z)\right)=\left\{q_{n}\right\}$ where $\partial O_{n}(z)$ stands for the boundary of $O_{n}(z)$ in $Y$. Such a choice of the map $h_{z}$ guarantees that $h_{z}(C \backslash\{z\})=\mathbb{I}$ for any nondegenerate subcontinuum $C \subset Y$ containing $z$.

Now consider the set $X=(Y \backslash Z) \cup(Z \times \mathbb{I})$ and the map $f: X \rightarrow Y$ which is the identity on $Y \backslash Z$ and $f(z, t)=z$ for each $(z, t) \in Z \times \mathbb{I} \subset X$. For every $z \in Z$ let $r_{z}: X \rightarrow\{z\} \times \mathbb{I}$ be a unique map such that 
- $r_{z}(y)=\left(z, h_{z}(y)\right)$ for every $y \in Y \backslash Z \subset X$;

- $r_{z}(y, t)=\left(z, h_{z}(y)\right)$ for every $(y, t) \in(Z \backslash\{z\}) \times \mathbb{I} \subset X$;

- $r_{z}(z, t)=(z, t)$ for every $t \in \mathbb{I}$.

Endow the space $X$ with the weakest topology making the maps $f: X \rightarrow Y$ and $r_{z}: X \rightarrow\{z\} \times \mathbb{I}, z \in Z$, continuous. According to [4, III.1.2] the resulting space $X$ is metrizable and compact. It is easy to check that the map $f$ is $I$-atomic (see also [4, III.1.15]).

Using the atomic property of $f$ and the Suslinian property of $Y$ it is easy to check that $X$ is Suslinian too.

Now, we are ready for the construction of our example. We note that similar constructions using atomic maps have been done before, for instance in [8], [10] and [11].

Theorem 6. Under the negation of the Suslin hypothesis there exists a hereditarily separable non-metrizable Suslinian continuum X. Moreover, each non-degenerate subcontinuum of $X$ is neither metrizable nor locally connected.

Proof. Assuming the negation of the Suslin hypothesis, fix a Suslin tree $(T, \leq)$ such that each node $t \in T$ has uncountably many successors in $T$ and infinitely many immediate successors in $T$. Denote by $h(t)$ the height of a node $t \in T$ and for a countable ordinal $\alpha$ let $T_{\alpha}=\{t \in T: h(t)=\alpha\}$ stand for the $\alpha$ th level of $T$. For two countable ordinals $\alpha<\beta$ let $\operatorname{pr}_{\alpha}^{\beta}: T_{\beta} \rightarrow T_{\alpha}$ denote the map assigning to a node $t \in T_{\beta}$ a unique node $t^{\prime} \in T_{\alpha}$ with $t^{\prime}<t$. We may additionally assume that the tree $T$ is continuous in the sense that for any limit countable ordinal $\alpha$ and distinct nodes $t, t^{\prime} \in T_{\alpha}$ there is $\beta<\alpha$ such that $\operatorname{pr}_{\beta}^{\alpha}(t) \neq \operatorname{pr}_{\beta}^{\alpha}\left(t^{\prime}\right)$.

We shall use transfinite induction to construct a well-ordered continuous spectrum $\left\{X_{\alpha}, \pi_{\alpha}^{\beta}: \alpha<\beta<\omega_{1}\right\}$ consisting of metrizable Suslinian continua $X_{\alpha}$ and atomic bonding maps $\pi_{\alpha}^{\beta}: X_{\beta} \rightarrow X_{\alpha}$, and a sequence $\left(i_{\alpha}: T_{\alpha} \rightarrow X_{\alpha}\right)_{\alpha<\omega_{1}}$ of injective maps such that

(1) for any countable ordinals $\alpha<\beta$ the diagram

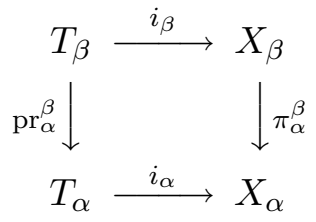

is commutative;

(2) for every $t \in T_{\alpha}$ the set $i_{\alpha+1}\left(\left(\operatorname{pr}_{\alpha}^{\alpha+1}\right)^{-1}(t)\right)$ is dense in $\left(\pi_{\alpha}^{\alpha+1}\right)^{-1}\left(i_{\alpha}(t)\right)$;

(3) the short projections $\pi_{\alpha}^{\alpha+1}: X_{\alpha+1} \rightarrow X_{\alpha}$ are $I$-atomic maps with non-degeneracy set $N\left(\pi_{\alpha}^{\alpha+1}\right)=i_{\alpha}\left(T_{\alpha}\right)$. 
We start the induction with a singleton $X_{0}$ and the injective map $i_{0}$ : $T_{0} \rightarrow X_{0}$ assigning to the root of $T$ the only point of $X_{0}$. Assume that for some countable ordinal $\alpha$ the Suslinian continua $X_{\beta}$, atomic bonding maps $\pi_{\gamma}^{\beta}: X^{\beta} \rightarrow X_{\gamma}$, and injective maps $i_{\beta}: T_{\beta} \rightarrow X_{\beta}$ have been constructed for all $\gamma \leq \beta<\alpha$.

If $\alpha$ is a limit ordinal, let $X_{\alpha}$ be the inverse limit of the countable spec$\operatorname{trum}\left\{X_{\beta}, \pi_{\gamma}^{\beta}: \gamma \leq \beta<\alpha\right\}$ and let $\pi_{\beta}^{\alpha}: X_{\alpha} \rightarrow X_{\beta}$ stand for the limit projections of this spectrum. They are atomic as limits of atomic bonding maps. For every $t \in T_{\alpha}$ let $i_{\alpha}(t)$ be the unique point of $X_{\alpha}$ such that $\pi_{\beta}^{\alpha}\left(i_{\alpha}(t)\right)=i_{\beta}\left(\operatorname{pr}_{\beta}^{\alpha}(t)\right)$ for every $\beta<\alpha$. The continuity of the tree $T$ implies that the resulting map $i_{\alpha}: T_{\alpha} \rightarrow X_{\alpha}$ is injective. The Suslinian property of $X_{\alpha}$ follows from that property of the continua $X_{\beta}, \beta<\alpha$, and the atomicity of the limit projections $\pi_{\beta}^{\alpha}$.

If $\alpha=\beta+1$ is a successor ordinal, then we can apply Lemma 3 to find a metrizable Suslinian continuum $X_{\alpha+1}$ and an $I$-atomic map $\pi_{\alpha}^{\alpha+1}$ : $X_{\alpha+1} \rightarrow X_{\alpha}$ whose non-degeneracy set coincides with $i_{\alpha}\left(T_{\alpha}\right)$. Thus we satisfy the condition (3) of the inductive construction. Since for every $t \in T_{\alpha}$ the set $\left(\pi_{\alpha}^{\alpha+1}\right)^{-1}\left(i_{\alpha}(t)\right)$ is an arc in $X_{\alpha+1}$, we can define an injective map $i_{\alpha+1}: T_{\alpha+1} \rightarrow X_{\alpha+1}$ so that $\pi_{\alpha}^{\alpha+1} \circ i_{\alpha+1}=i_{\alpha} \circ \operatorname{pr}_{\alpha}^{\alpha+1}$ and $i_{\alpha+1}$ satisfies the condition (2) of the inductive construction.

After completing the inductive construction, consider the inverse limit $X$ of the spectrum $\mathcal{S}=\left\{X_{\alpha}, \pi_{\beta}^{\alpha}: \beta<\alpha<\omega_{1}\right\}$. Using the atomicity of the bonding projections, one can check that the limit projections $\pi_{\alpha}: X \rightarrow X_{\alpha}$ are atomic as well.

Now, we establish the desired properties of the continuum $X$. First, we show that each non-degenerate subcontinuum $C$ of $X$ is neither metrizable nor locally connected. Let $\alpha$ be the smallest ordinal such that $\left|\pi_{\alpha}(C)\right|>1$. The continuity of the spectrum $\mathcal{S}$ implies that $\alpha=\beta+1$ for some ordinal $\beta$. Then $\pi_{\beta}(C)$ is a singleton and hence $\pi_{\beta}(C) \subset i_{\beta}\left(T_{\beta}\right)$ (otherwise $C$ would be a singleton). Let $t \in T_{\beta}$ be a node of $T$ with $\pi_{\beta}(C)=\left\{i_{\beta}(t)\right\}$. It follows that $\pi_{\alpha}(C)$ is a non-degenerate subcontinuum of the $\operatorname{arc} A_{t}=\left(\pi_{\beta}^{\alpha}\right)^{-1}\left(i_{\beta}(t)\right)$. The density of $i_{\alpha}\left(T_{\alpha}\right)$ in $A_{t}$ implies the existence of a node $t^{\prime} \in T_{\alpha}$ with $i_{\alpha}\left(t^{\prime}\right) \in$ $\pi_{\alpha}(C)$. The atomicity of the projection $\operatorname{pr}_{\alpha}$ implies that the continuum $C=$ $\pi_{\alpha}^{-1}\left(\pi_{\alpha}(C)\right)$ contains the subcontinuum $\operatorname{pr}_{\alpha}^{-1}\left(i_{\alpha}\left(t^{\prime}\right)\right)$ which is not metrizable (because $t^{\prime}$ has uncountably many successors in the tree $T$ ). Consequently, $C$ is not metrizable either.

To show that $C$ is not locally connected, assume the converse and, given any two distinct points $x, x^{\prime} \in \mathrm{pr}^{-1}(\alpha)\left(i_{\alpha}\left(t^{\prime}\right)\right)$, find a closed connected neighborhood $U \subset C$ of $x$ with $x^{\prime} \notin U$. Since $\operatorname{pr}_{\alpha}^{-1}\left(i_{\alpha}\left(t^{\prime}\right)\right)$ is nowhere dense in $C$, the set $U$ has non-degenerate projection $\operatorname{pr}_{\alpha}(U)$. Then the atomicity of $\operatorname{pr}_{\alpha}$ implies that $x^{\prime} \in \operatorname{pr}_{\alpha}^{-1}\left(\operatorname{pr}_{\alpha}(U)\right)=U$, which is a contradiction. 
Next, we shall prove that the continuum $X$ is Suslinian. Take any family $\mathcal{C}$ of pairwise disjoint non-degenerate subcontinua in $X$. Repeating the preceding argument, for every $C \in \mathcal{C}$ we can find a countable ordinal $\alpha$ and a node $t_{C} \in T_{\alpha}$ such that $C \supset \pi_{\alpha}^{-1}\left(i_{\alpha}\left(t_{C}\right)\right)$. It follows that the nodes $t_{C}, C \in \mathcal{C}$, are pairwise incomparable in $T$ (otherwise the family $\mathcal{C}$ would contain two intersecting continua). Since $T$ is a Suslin tree, the antichain $\left\{t_{C}: C \in \mathcal{C}\right\}$ is at most countable and so is the family $\mathcal{C}$, witnessing the Suslinian property of $X$.

It remains to check that the continuum $X$ is hereditarily separable. By $[3,3.12 .9]$ it suffices to prove that each closed subspace $F$ of $X$ in separable. By Theorem 2, the continuum $X$, being Suslinian, is perfectly normal and hence $F=\pi_{\alpha}^{-1}\left(\pi_{\alpha}(F)\right)$ for some countable ordinal $\alpha$. Let $Z=\operatorname{pr}_{\alpha}(F)$. Since

$$
F=\pi_{\alpha}^{-1}\left(Z \backslash i_{\alpha}\left(T_{\alpha}\right)\right) \cup \bigcup_{z \in Z \cap i_{\alpha}\left(T_{\alpha}\right)} \pi_{\alpha}^{-1}(z)
$$

and $\pi_{\alpha}^{-1}\left(Z \backslash i_{\alpha}\left(T_{\alpha}\right)\right)$ is homeomorphic to the metrizable separable space $Z \backslash i_{\alpha}\left(T_{\alpha}\right)$, it remains to check that for every $z \in i_{\alpha}\left(T_{\alpha}\right)$ the continuum $\pi_{\alpha}^{-1}(z)$ is separable. Consider the $\operatorname{arc} A=\pi_{\alpha}^{\alpha+1}(z)$ in $X_{\alpha+1}$ and observe that $D=A \backslash i_{\alpha+1}\left(T_{\alpha+1}\right)$ is a dense subspace of $A$. It follows from the construction that $\pi_{\alpha+1}^{-1}(D)$ is a topological copy of $D$, dense in $\pi_{\alpha+1}^{-1}(A)=$ $\pi_{\alpha}^{-1}(z)$. Therefore, the continuum $\pi_{\alpha}^{-1}(z)$ is separable.

We do not know if the preceding theorem can be generalized to higher cardinals.

Problem 2. Does the existence of a $\kappa^{+}$-Suslin tree imply the existence of a continuum $X$ with $h d(X) \leq \operatorname{Sln}(X)=\kappa<w(X)$ ?

REMARK 1 . The existence of a $\kappa^{+}$-Suslin tree is equivalent to the existence of a linearly ordered continuum $X$ with $\kappa=\operatorname{Sln}(X)=c(X)<d(X)=$ $w(X)=\kappa^{+}$.

The non-metrizable hereditarily separable Suslinian continuum constructed in Theorem 6 is very far from being locally connected. In [2], it was proved that separable homogeneous Suslinian continua are metrizable. This encourages us to recall the following question of [1].

Problem 3. Is each locally connected (hereditarily) separable Suslinian continuum metrizable?

Acknowledgments. The fourth named author is partially supported by National Science and Engineering Research Council of Canada grants No. 141066-2009. 


\section{References}

[1] D. Daniel, J. Nikiel, L. Treybig, M. Tuncali and E. D. Tymchatyn, On Suslinian continua, Canad. Math. Bull. 48 (2005), 195-202.

[2] - , - - - - - - Homogeneous Suslinian continua, Canad. Math. Bull., to appear.

[3] R. Engelking, General Topology, PWN, Warszawa, 1977.

[4] V. V. Fedorchuk, Fully closed mappings and their applications, J. Math. Sci. 136 (2006), 4201-4292.

[5] V. V. Fedorchuk and A. Chigogidze, Absolute Retracts and Infinite-Dimensional Manifolds, Nauka, Moscow, 1992.

[6] K. Kuratowski, Topology, Volume II, PWN, 1968.

[7] A. Lelek, On the topology of curves I, Fund. Math. 67 (1970), 359-367.

[8] T. Maćkowiak, Singular arc-like continua, Dissertationes Math. 257 (1986), 40 pp.

[9] S. Mardešić, Locally connected, ordered and chainable continua, Rad Jugoslav. Akad. Znan. Umjet. Odjel Mat. Fiz. Tehn. Nauke 319 (1961), 147-166.

[10] L. Mohler and L. G. Oversteegen, On the structure of tranches in continuously irreducible continua, Colloq. Math. 54 (1987), 23-28.

[11] - - - On hereditarily decomposable hereditarily equivalent nonmetric continua, Fund. Math. 136 (1990), 1-12.

[12] S. Nadler, Continuum Theory, Dekker, 1992.

T. Banakh

Uniwersytet Humanistyczno-Przyrodniczy

Jana Kochanowskiego

Kielce, Poland

and

Department of Mathematics

Ivan Franko Lviv National University

Lviv, Ukraine

E-mail: tbanakh@yahoo.com

J. Nikiel

Instytut Matematyki i Informatyki

Uniwersytet Opolski

Oleska 48

45-052 Opole, Poland

E-mail: nikiel@math.uni.opole.pl
V. V. Fedorchuk Faculty of Mechanics and Mathematics Lomonosov Moscow State University Vorob'evy Gory, 1

Moscow, Russia E-mail:vvfedorchuk@gmail.com

M. Tuncali Nipissing University North Bay, Ontario, Canada E-mail: muratt@nipissingu.ca

Received 24 March 2009;

in revised form 15 January 2010 